Proceedings of the 2010 Winter Simulation Conference

B. Johansson, S. Jain, J. Montoya-Torres, J. Hugan, and E. Yücesan, eds.

\title{
A SIMULATION APPROACH TO ESTIMATE THE VALUE OF INFORMATION IN MARITIME SUPPLY CHAINS
}

\author{
U. Hari Prasad \\ Convenor's Office \\ Industrial Engineering and Operations Research Programme \\ IIT Bombay, Powai, Mumbai 400076, India
}

\author{
Srinagesh Gavirneni \\ Johnson Graduate School of Management \\ Cornell University \\ Ithaca, NY 14853, USA
}

\begin{abstract}
With the rapid increase in global trade and introduction of new security measures, maritime supply chain costs have increased and so has the need for business intelligence in improving maritime shipping operations. We develop a seaport operations model that simulates the decision making process associated with scheduling and processing of ships with the objective of evaluating the value of Geographical Information System (GIS) information. We consider two scenarios: (1) A traditional model where there is no GIS information on future ship arrivals; and (2) An information-rich model in which the arrival time of the next ship is known. We propose look-ahead based heuristics for the resulting optimization problems, determine the value of information (VOI), and tabulate how VOI varies as a function of the various operational parameters. Adding such operational intelligence to shipping operations improves the performance by as much as $60 \%$ (and by $15 \%$ on average) and reduces the costs without expanding the physical footprint of the seaport.
\end{abstract}

\section{INTRODUCTION}

Transportation by sea is an important part of the modern global supply chains and any delays at ports result in increased costs to the end-consumers. Modeling, analysis, and management of maritime supply chains are vital to keep the world economic engine humming. Delays in cargo processing (loading and unloading of ships) could be detrimental to port efficiency, thereby impacting not only their competitiveness, but also the competitiveness of the supply chains they are part of. Congestion resulting from poor management of seaports can also affect shipping lines, manufacturers, distributors, freight forwarders, warehouses, consumers and everyone else along the supply chain. It causes ripple effects throughout the nation's (and even world's) economy and disrupts Just-In-Time inventory practices now commonly employed by most companies. Manufacturers and service providers, in most cases, pass on the cost incurred through the delay of their raw materials and equipment at the ports, to the consumers, leading to higher prices for goods and services.

It goes without saying that the problem of port congestion could adversely affect the economic competitiveness of trade dependent nations. Given the gravity of the issue, strategic decision making and business intelligence become extremely important for terminal operators and port authorities so that they can improve the efficiency of port operations. The operational performance of a port is generally measured in terms of the speed with which a vessel is dispatched, the rate at which cargo is handled, and the duration that cargo stays in port prior to shipment or post discharge. There is a need to develop strategies that help the ports operate efficiently along all these dimensions.

One of the most promising solutions to this issue is the integration of Geographical Information Systems (GIS) with the port decision making systems. Coupled with external databases and sound analytical tools, this constitutes the input for an internal spatiotemporal database that manages and analyzes historical and real-time data of ships. These data include maritime trajectories, arrival times, vessel characteristics, and environmental information. A GIS can recognize and analyze the spatial relationships that exist within digitally stored spatial data. These topological relationships allow complex spatial modeling and associated analysis can lead to improved decision making.

In this paper, we develop simulation and analytical models of a typical seaport in order to determine how the GIS data can be used in a business intelligence framework to improve the decision making process. While the operations at a seaport are, in reality, very complex and that no mathematical model can capture all their aspects, we focus on what we feel are the most important characteristics. We specifically focus on the fact that a seaport operator has the flexibility 


\section{Prasad and Gavirneni}

to increase the speed (at an additional cost) at which a ship is serviced so as to minimize the waiting costs associated with the other ships. This decision on whether or not to expedite the current ship would depend on the arrival times, and the importance of the ships scheduled to come in the future. The aforementioned GIS database will provide that information and one can use it to optimize system performance which we model here as the sum of expediting and waiting costs. We use the mathematical analysis and the simulation model to determine the extent of benefit that can be recognized from such a GIS database and also characterize the role of various system parameters in maximizing this benefit.

\section{MODEL SETUP}

We consider the long term operation of seaport that is receiving and processing a series of ships. The port is modeled as a multi-server queuing system with a stream of incoming ships. The time required to process/service a ship is randomly distributed with a known distribution. The arrival process of the ships is stochastic with a known distribution of inter-arrival times. There are two modes (namely, regular and expedited) of processing available for any ship. Without loss of generality, we assume that regular processing costs nothing while the expedited service costs $c_{e}$ dollars. We assume that when the expedited service mode is invoked, the time to service the ship drops from $t$ to $0.5 t$. The key decision in this study is the one associated with whether or not a ship should be expedited. We denote the decision by $d_{i} \in\{0,1\}$ for ship $i$ and $d_{i}=1$ if the ship is indeed expedited. These decisions are made to minimize the total costs associated with ships waiting to be serviced by the port. We denote by $w(i, t)$, the cost incurred when ship $i$ waits for $t$ time units. Through most of this paper, we will assume that this cost is linear in waiting time.

In order to facilitate a detailed analysis of this queuing system, we make a number of assumptions (most are reasonable and can be justified in practice) which are detailed below:

- Ships are processed on a First Come First Served (FCFS) basis

- Preempting is not allowed. That is, once the processing of a ship is started, it cannot be temporarily halted in order to service another (probably more important) ship.

- The mode (regular or expedited) of service cannot be changed once the service has commenced.

- Information on arrival times of future ships, if available, is perfectly accurate. That is, there is no further randomness and there are no errors in creation and transmission of this information.

- Ships can be serviced continuously without a need for downtime between ships.

- The server is perfectly reliable and is available $100 \%$ of the time.

Many of the these assumptions, we believe, can be relaxed without significantly affecting the simulation model and the results. Given this model setup, the problem can be stated as determine the expediting sequence, $d_{1}, d_{2}, d_{3} \ldots$, of ships so that the sum of expediting and waiting costs is minimized.

We can represent each ship by a node in a graph and the two possible decisions as two edges leading to the next ship/node. Then the decision making process can be represented by a binary decision tree, where any path from the root node to a leaf node is a possible expediting scheme and a feasible solution to the problem. Figure 1 shows the graphical representation an $\mathrm{N}$-level tree with the regular mode of service on the right and the expedited mode of service on the left. The objective is to determine the lowest cost path from the root node to a leaf node. We model

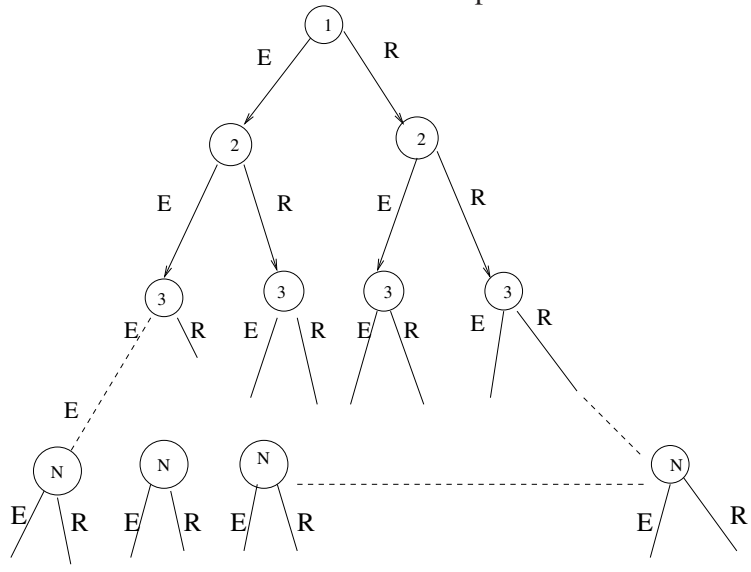

Figure 1: Decision Tree associated with $N$ ships

two informational scenarios when the decision at the root node is being made. 


\section{Prasad and Gavirneni}

- Scenario NI: No information is available on the arrival times of the future ships.

- Scenario A1: Arrival time of the next ship is known.

We develop solutions for solving the two problems that arise from these scenarios. It is possible to model scenario $\mathrm{A} n$, in which the arrival times of the next $n$ ships are known. The analysis associated with An is quite complex and we leave that extension as a possibility for future research.

\section{SOLUTION PROCEDURES}

In this section we develop approaches to solve the aforementioned decision problems. Once these procedures have been elucidated, we describe a simulation methodology that we used to implement these solution procedures and eventually report on the cost reductions that can be attributed to the availability of information.

\subsection{SCENARIO NI}

Under this scenario when the decision at the root node is being made, no information is available about future arrivals. However the probability distributions of arrival times and the service times are known from which the average utilization of the server could be computed. When this utilization is low, it would be best for the server to always operate in the regular mode and when the utilization is high, it would be best for the server to always operate in the expedited mode. While it would be possible to analytically derive (at least for a few specific distributions) the threshold value at which this switch occurs, we decided to simulate both policies and choose the one with lower cost. In summary, for scenario NI, we simulate the system under (i) always-regular operating policy; and (ii) always-expedited operating policy. We then tabulate the costs under the two policies, and choose the strategy with the lower cost.

\subsection{SCENARIO A1}

For this informational scenario, we propose a look-ahead procedure for making decisions on whether or not to expedite the incumbent ship. Let us assume that this decision is being made at time $t$ and that the incumbent ship is ship $i$ with regular processing time of $p_{i}$. Given that we are in scenario A1, the arrival time, $a_{i+1}$ is known. If $t+p_{i}$ is smaller than $a_{i+1}$, then there is no interaction between ships $i$ and $i+1$ and it is optimal to process ship $i$ at the regular speed. On the other hand, if $t+p_{i}$ is greater than $a_{i+1}$, and if ship $i$ is processed at the regular mode, then ship $i+1$ will incur a waiting cost. The magnitude of this waiting cost will play a critical role in determining whether or not ship $i$ should be served in an expedited manner. We have to balance this incurred waiting cost with the cost of expediting and to do that we introduce a parameter and designate that ship $i$ should be expedited if $t+p_{i}->a_{i+1}$.

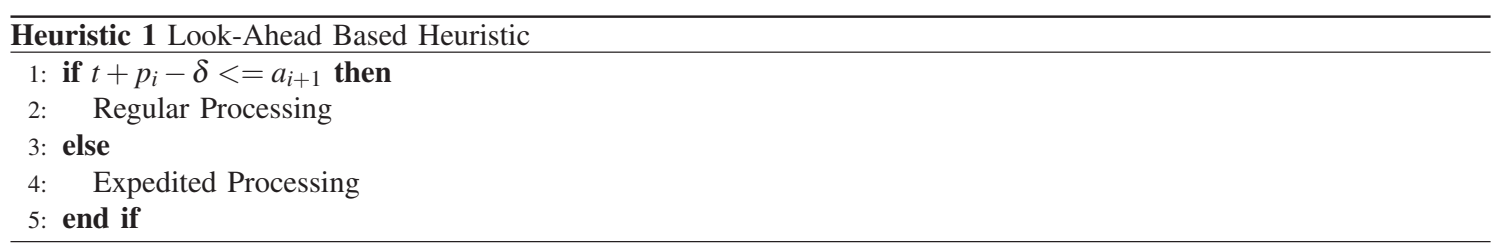

The parameter must be chosen carefully so that sum of the expediting costs and waiting costs is minimized. If the waiting costs dominate the expediting costs, then the optimal value would be smaller (i.e. expediting is invoked often) and when the expediting costs dominate the waiting costs, then the optimal value would be larger (i.e. expediting is not invoked often). Using simulation, we search over various possible values of and pick the one that results in the lowest cost.

\section{SIMULATION MODEL}

We built a simulation model that captures the characteristics described earlier. Needless to say, this system is not that different from a multi-server queuing system and thus our simulation is similar to ones described in earlier literature. The process begins when a ship arrives at the port. If the processing unit is free, then the ship will be granted permission to enter the berth. If the berth is not available, then the ship will be delayed incurring a waiting cost. When a ship enters the berth, then a decision has to be made about whether that ship will be served in an expedited manner. That decision will be made according to the algorithms described earlier for each information scenario. The server becomes idle waiting for the next ship to arrive if the queue is empty when a ship completes processing. On the other hand, if the queue is not empty, then the first ship (recall the FCFS service assumption) in the queue is brought into the berth and expediting decision is once again made. 
The model assumes a normal distribution for ship inter-arrival times with mean $\quad{ }_{a}$ and variance $\quad{ }_{a}$ and for processing times with mean $\quad p$ and variance $p$. We model different values of waiting cost $c_{w}$ (dollars per hour) and expediting $\operatorname{cost} c_{e}$ (dollars).

\subsection{Scheduling Procedures}

We formally describe algorithmic procedures that we implemented in order to make decisions about which ships should be expedited so as to minimize the total costs.

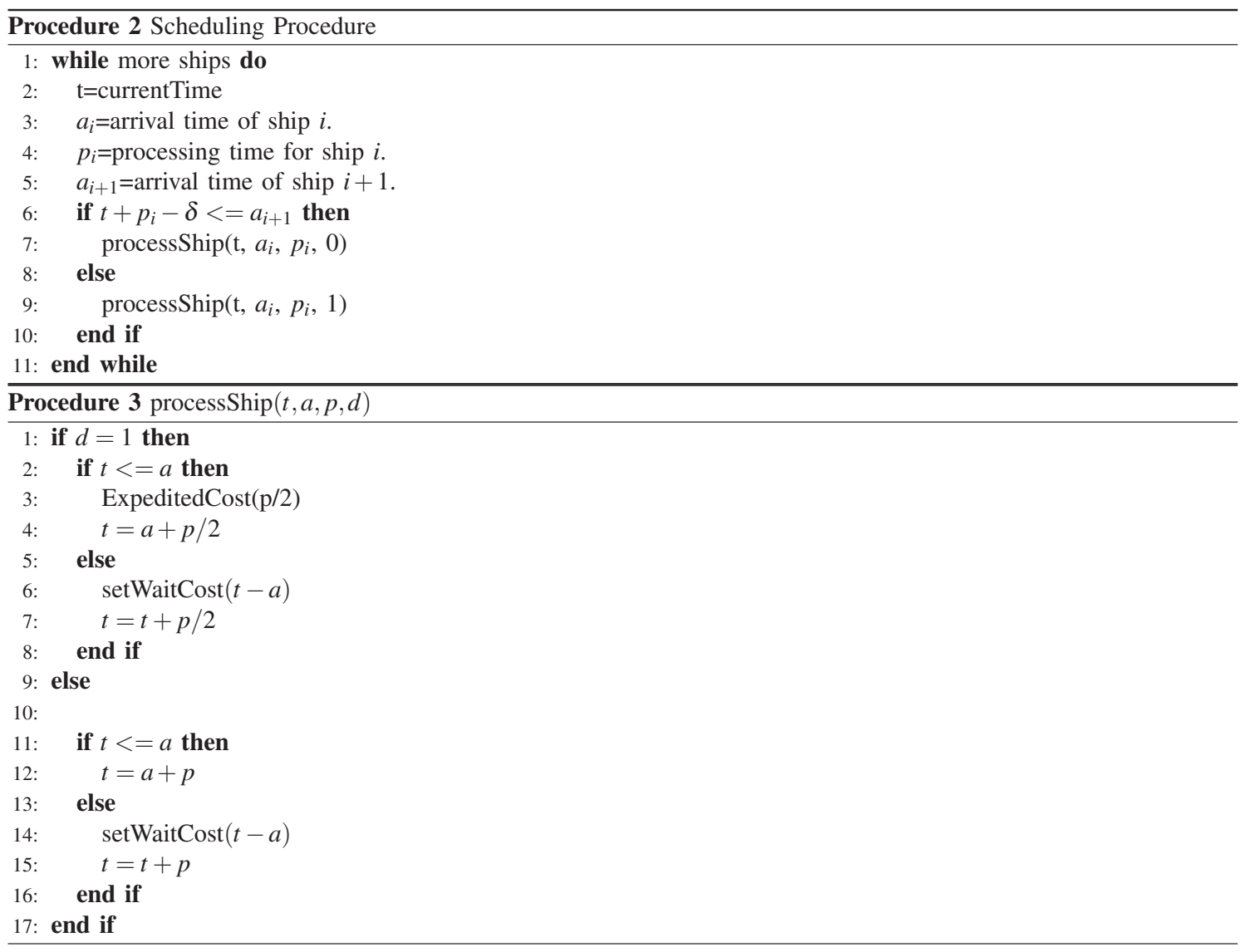

\section{VALUE OF INFORMATION}

Under scenario NI, all ships are either processed at the regular speed or at the expedited speed. Let $T_{r}$ be the total cost at regular-always processing and $T_{e}$ the total cost of expedited-always processing. Under scenario A1, we use the look-ahead policy and let $T_{o}$ be the total cost under this strategy. We compute the value of information (percentage reduction in cost) as:

$$
\% V O I=\frac{\min \left(T_{r}, T_{e}\right)-T_{o}}{\min \left(T_{r}, T_{e}\right)} \times 100
$$

Now that we have the ability to compute the value of information, we are interested in answering the following questions about its behavior:

1. What is the magnitude of the percentage value of information across a wide range of parameters?

2. Is the value of information larger when the system is less congested or more congested?

3. How does uncertainty (in arrival and service processes) affect the magnitude of VOI?

4. What is the role of relationship between expediting and waiting costs in determining the value of information?

5. How does the number of servers impact the percentage value of information? 


\section{Prasad and Gavirneni}

To answer these questions, we designed a comprehensive experimental study and ran the simulations, tabulated the observed values of information, and analyzed them for trends. In the study, values were assigned to the parameters as follows: The inter-arrival times have means of $8,10,12,14$, and 16 hours while they have coefficients of variance of $0.05,0.10,0.15,0.20$, and 0.25 . The processing times have means of $6,7.5,9,10.5$, and 12 hours while they have coefficients of variance of $0.05,0.10,0.15,0.20$, and 0.25 . The waiting costs are $2,4,6,8,10$ dollars per hour while the expediting costs are $25,50,75,100$, and 125 . Thus we have a total 15,625 instances that we use to derive our managerial insights.
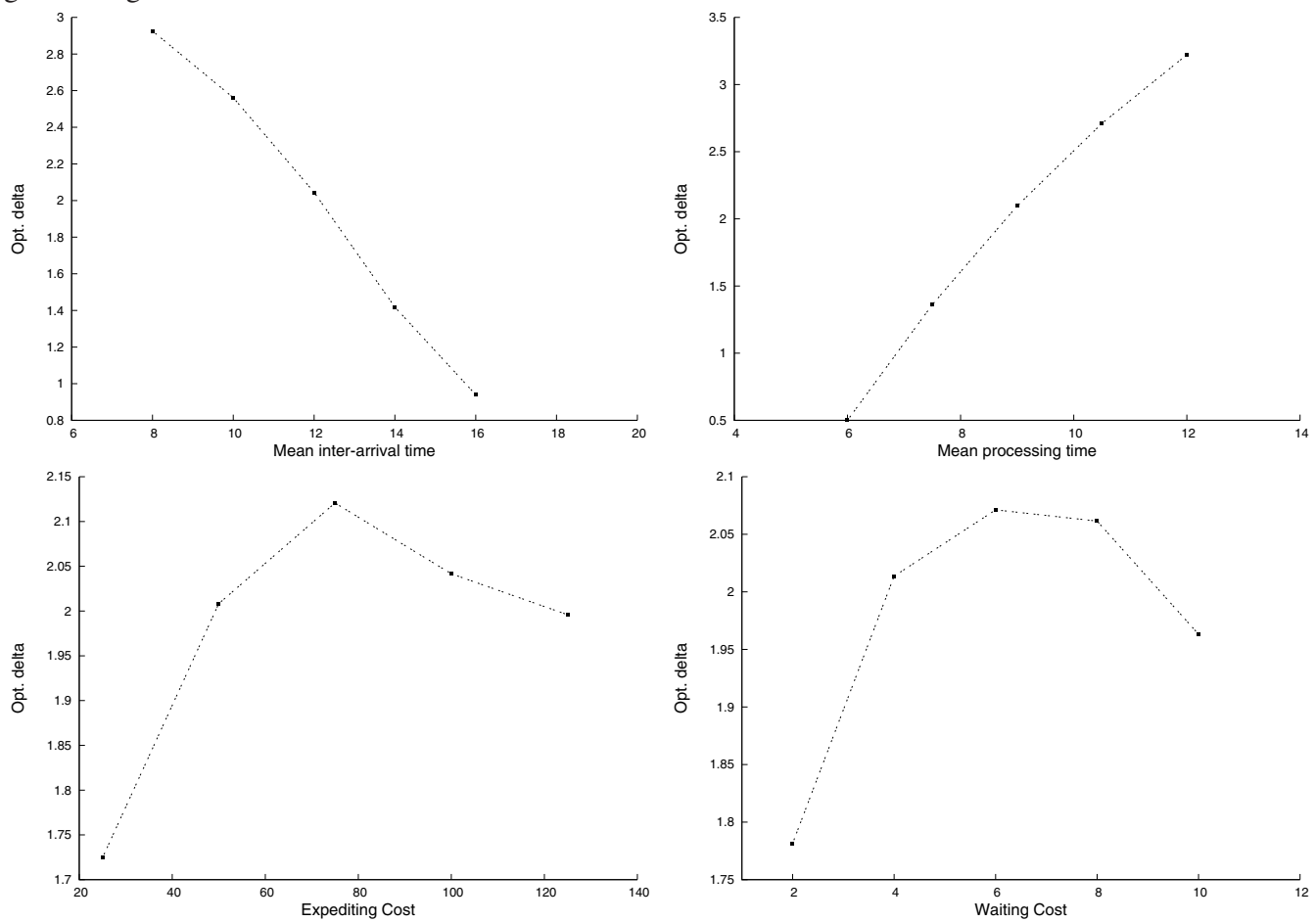

Figure 2: Behavior of optimal with respect to system parameters

\subsection{Behavior of}

Before we present the results on value of information, we describe how the parameter changes with respect to the various system parameters. Notice from the top portion of Figure 2 that as mean inter-arrival time increases, the value decreases and that as the mean processing time increases, the value increases. At higher (lower) values of inter-arrival (processing) time, the probability that two adjacent ships will conflict is lower and thus there is reduced need for expediting. As a result, the optimal value is very often zero and thus the average optimal is lower. When the system has lower (higher) values of inter-arrival (processing) time, then there is a need to actively decide on which ships to expedite and to do that the value that best balances expediting and waiting costs must be chosen. This value is often different from zero and thus the average optimal value is larger under those settings.

The bottom half of Figure 2 shows that the optimal value first increases as the expediting and waiting costs increase and then eventually decreases. When expediting cost is small and/or the waiting cost is large, then all the ships should be expedited resulting in the optimal value being smaller. At higher expediting costs and lower waiting costs, the frequency of expediting decreases implying that the values should be set higher. As the expediting and waiting costs increase further, the system stabilizes at an optimal value, which is necessary to achieve a balance between waiting and expediting needed to minimize the cost of shipping operations.

\subsection{Value of Information}

In this section, we focus on the value of information observed in the 15,625 instances we studied in the simulation. We observed that it was as large as $60 \%$ and was about $15 \%$ on the average. To understand how the various system parameters affect the value of information, we performed a detailed analysis of the results. For each value of a system 


\section{Prasad and Gavirneni}

parameter, we computed the average percentage VOI observed over the all instances with that value for the parameter. This enabled us to evaluate the trends that \% VOI displays with respect to the various system parameters.

\subsubsection{Impact of Inter-arrival and Processing Times}

The top half of Figure 3 captures the relationship between \% VOI with respect to mean inter-arrival time and mean processing time. Notice from the figure that as the inter-arrival time increases, the percentage VOI decreases. On the other hand, as the mean processing time increases, the \% VOI increases. Further analysis shows that \% VOI is largest when the ratio of inter-arrival and processing times is close to 1 implying that when the arrival and service processes are perfectly matched on average, it is necessary to use the information to overcome the uncertainty in the system. When the ratio is much smaller than 1 , then all the ships will be processed in an expedited manner (just to keep the system balanced) and when the ratio is much larger than 1, then the expedited mode will never be invoked. That is, in those two extreme cases, there is no value to the information on future arrivals. Thus, we conclude that a seaport must consider investing in a GIS database when it has utilization, under regular speed of processing, close to $100 \%$.

\subsubsection{Impact of Expediting and Waiting Costs}

The bottom half of Figure 3 displays the relationship between \% VOI and expediting and waiting costs. Notice that as expediting cost increases, the \% VOI decreases and that as the waiting cost increases, the \% VOI increases. When expediting cost is relatively large, then the expediting mode will never be invoked and as a result the information about the arrival times of future ships will not be that valuable. On the other hand, when the waiting cost is relatively large, then it is important to actively manage the expediting process and thus the information about future arrivals is immensely valuable. Thus a seaport should invest in a GIS database when the waiting costs are larger (at least in a relative sense) than expediting costs.
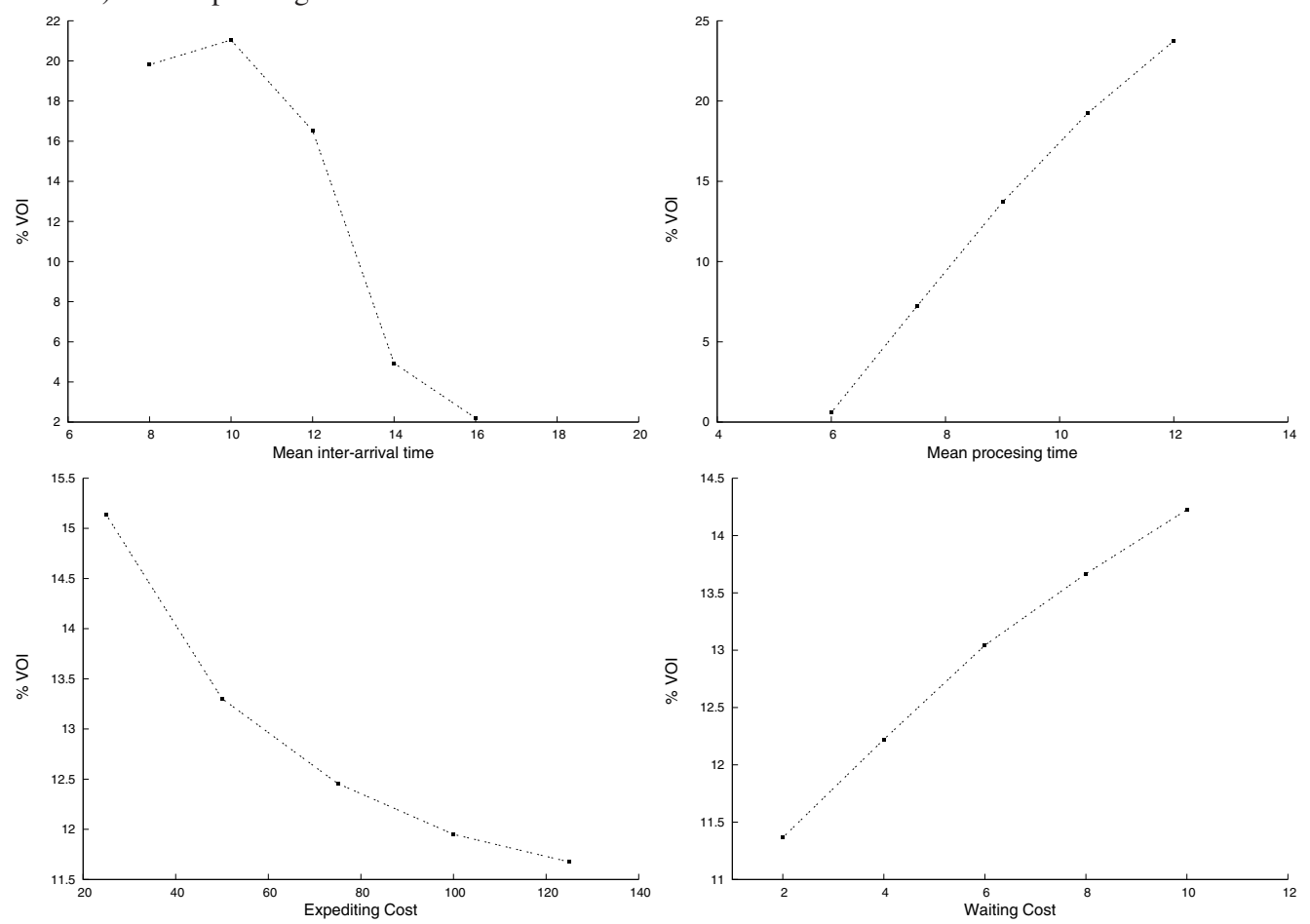

Figure 3: Percentage VOI as a function of the various system parameters

\subsubsection{Impact of Inter-arrival Time Variance and Processing Time Variance}

Figure 4 displays the trend in percentage value of information as the variance of inter-arrival times and processing times increase. Observe that when the system has a higher degree of uncertainty the information is more valuable. This is because the uncertainty plays a critical role in determining the interaction between waiting costs and expediting costs. When these interactions are severe, one needs to be careful in making the expediting decisions and information on 

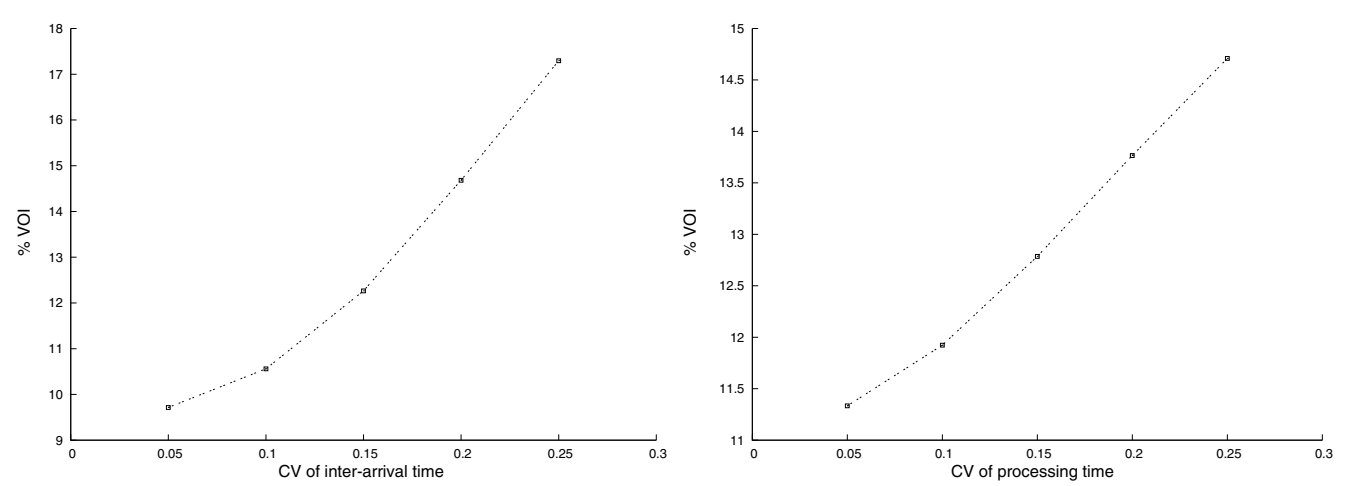

Figure 4: Percentage VOI as a function of (i) coefficient of variation of inter-arrival time; and (ii) coefficient of variation of processing time.

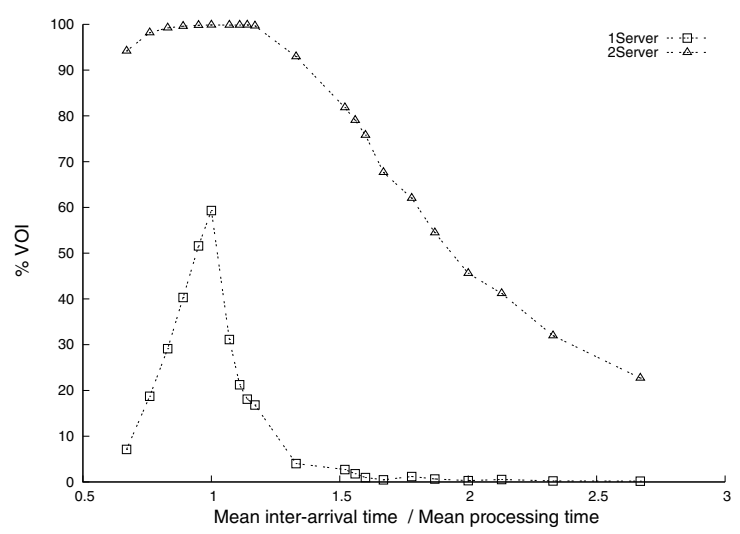

Figure 5: Percentage VOI observed in the presence of one server and two servers.

future arrivals can help make better decisions. As a result, we conclude that a seaport should invest in a GIS database when the system has considerable uncertainty in arrival and processing times.

\section{MULTI-SERVER SYSTEM}

In this section, we extend the analysis presented above to accommodate the presence and availability of multiple servers. We adapt the look-ahead procedure described in earlier sections to account for the fact that the expediting decisions are now more complicated. The generalized algorithm for $s$ servers is given below. We implemented this procedure and performed an analysis comparing the percentage value of information in presence of one server and two servers. The results are demonstrated in Figure 5. Observe that, while the trend is similar across these two situations, the percentage VOI observed is much larger in the presence of two servers. The presence of two servers increases the flexibility the system possesses and this additional flexibility enables the port to better use the available information resulting in the larger reduction in cost. Thus, we conclude that the investment in a GIS database is more attractive when the port is being serviced my multiple servers.

\section{CONCLUSION AND FUTURE RESEARCH}

We modeled a typical seaport as a queuing system in order to study when and how the expediting option (available at an additional cost) should be exercised in order to reduce or minimize waiting costs. We developed an analytical framework and coupled that with a detailed simulation study to draw managerial insights on when the investment in a GIS database should be made in order to improve the efficiency of the seaport operation. We concluded that such an investment should be made when the system utilization is neither too large nor too small, the uncertainty is high, and the waiting cost is larger (in a relative sense) than expediting cost. 


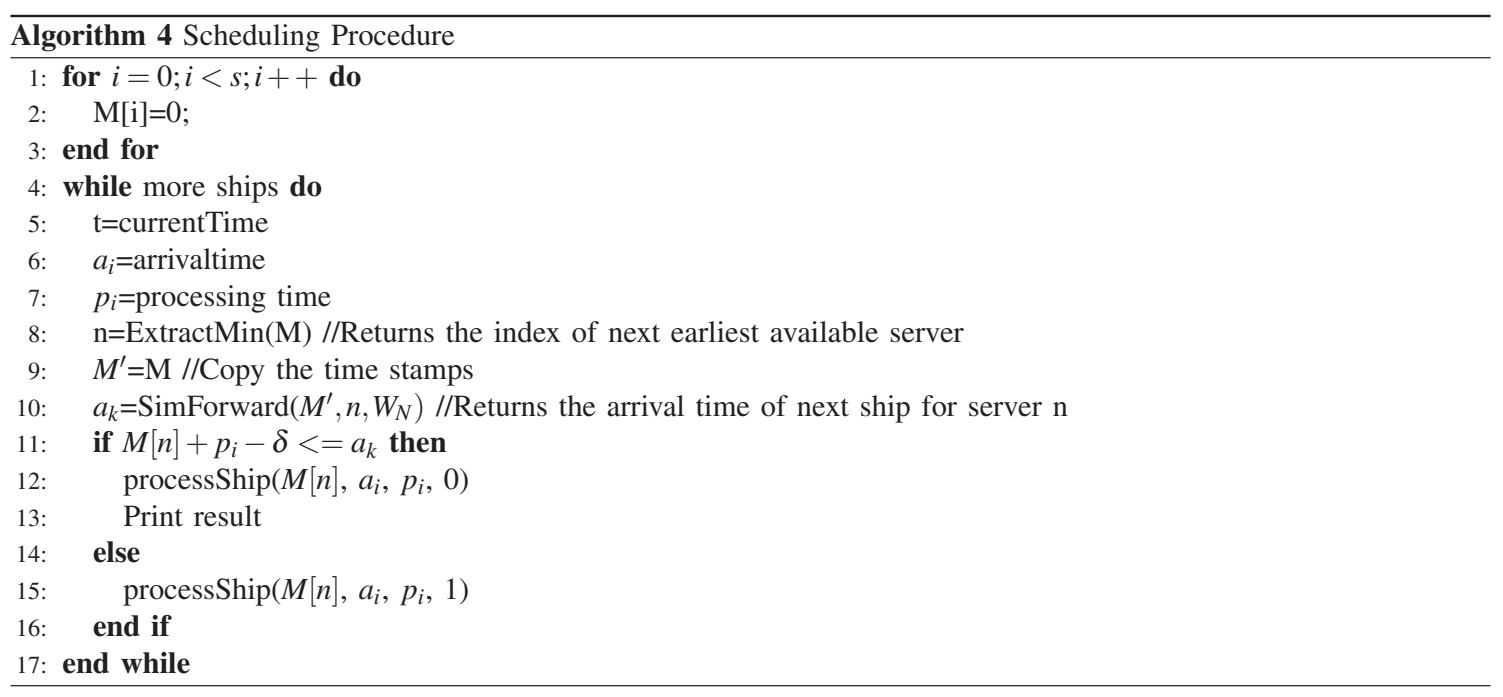

There are a number of directions in which this research can be extended. The multi-server system which we were able to study here only in a superficial fashion deserves a much more detailed scrutiny and analysis. Another direction for future research is to incorporate the possibility that ships in transit can be either sped up (at additional cost) or slowed down (at substantial savings) and these decisions can be made in conjunction with the expediting decision so that the total system costs are minimized. Finally, the seaport can be incorporated into a larger supply chain setting so that the impact of these decisions on the global economy can be better understood.

\section{REFERENCES}

Camm, J. D., T. E. Chorman, F. A. Dill, J. R. Evans, D. J. Sweeney, and G. W. Wegryn. 1997. Blending or/ms, judgment, and gis: Restructuring p\&g's supply chain. INFORMS 27:128-142.

Gavirneni, S., R. Kapunscinski, and S. Tayur. 1999. Value of information in capacitated supply chains. Management Science 45:16-24.

Guo, X., S. Y. Huang, W. J. Hsu, and M. Y. H. Low. 2008. Yard-crane dispatching based on real time data driven simulation for container terminals. In Proceedings of the 2008 Winter Simulation Conference, ed. S.J. Mason, R.R. Hill, L. Monch, O. Rose, T. Jefferson and J.W. Fowler, 2648-2655. Piscataway, New Jersey: Institute of Electrical and Electronics Engineers, Inc.

Kozon, E. 1997. Comparison of analytical and simulation planning models of seaport conatiner terminals. Transportation Planning and Technology 20:235-248.

Nevins, M. R., C. M. M. Joseph, and C. Jones. 1998. Discrete-event-simulation model for seaport operations. SIMULATION 70 (4): 213-223.

Sasso, D., and W. E. Biles. 2008. An object-oriented programming approach for a gis data-driven simulation model of traffic on an inland waterway. In Proceedings of the 2008 Winter Simulation Conference, ed. S.J. Mason, R.R. Hill, L. Monch, O. Rose, T. Jefferson and J.W. Fowler, 2590-2594. Piscataway, New Jersey: Institute of Electrical and Electronics Engineers, Inc.

Tahar, R. M., and K. Hussain. 2000. Simulation and analysis for the kelang seaport terminal. Logistics Information Management 13:14-20.

Whitt, W. 1992. Understanding the efficiency of multi-server service systems. Management Science 38:708-723.

\section{AUTHOR BIOGRAPHIES}

U. HARI PRASAD is an operations software consultant in India. His interests are in the areas of optimization, sequencing and scheduling, high-performance computing and verification. He has worked in the semiconductor industry for four years with market leaders like Xilinx and Cadence and has architected, developed and maintained production level softwares in the areas of logic verification, synthesis and simulation. He is now consulting with Indian Railways on investment analysis and rail infrastructure planning. He holds a bachelor degree in Computer Science and Engineering from Indian Institute of Technology - Bombay.

SRINAGESH GAVIRNENI is an associate professor of operations management in the Johnson Graduate School of Management at Cornell University. His research interests are in the areas of supply chain management, inventory control, production scheduling, simulation, and optimization. His papers have appeared in Management Science, 


\section{Prasad and Gavirneni}

Manufacturing \& Service Operations Management, Operations Research, European Journal of Operational Research, Operations Research Letters, IIE Transactions, and Interfaces. Previously, he was an assistant professor in the Kelley School of Business at Indiana University, the chief algorithm design engineer of SmartOps, a software architect at Maxager Technology Inc., and a research scientist with Schlumberger. His undergraduate degree from Indian Institute of Technology - Madras is in mechanical engineering, and he has received an M.Sc. from Iowa State University and a $\mathrm{Ph} . \mathrm{D}$. from Carnegie Mellon University. 